\title{
On the Hurewicz image of the cokernel $J$ spectrum
}

\author{
by \\ Norihiko Minami (Tuscaloosa, Ala.) \\ Dedicated to Professor Mark Mahowald on the \\ occasion of his retirement from teaching calculus
}

\begin{abstract}
We prove that the integral Hurewicz image of the cokernel $J$ spectrum detects precisely the Kervaire invariant one elements and nothing else, which may be viewed as an analogue of the Curtis-Madsen conjecture on the unstable mod 2 Hurewicz image of $Q_{0} S^{0}$.

1. Introduction. Ever since Browder's paper [Bro], the Kervaire invariant one problem $[\mathrm{Ker}],[\mathrm{KM}]$ has been very influential in homotopy theory (see [Mah2], [BJM1], [BJM2], [Min2], [Min4] for instance). One of the related problems is the Curtis-Madsen conjecture [Cur], [Mad] which predicts that the mod 2 unstable Hurewicz map of $Q_{0} S^{0}$ detects precisely the Hopf invariant one elements, the Kervaire invariant one elements, and nothing else. However, the Curtis-Madsen conjecture still appears to be an extremely difficult problem, as is the Kervaire invariant problem itself (cf. [Wel]).

Now the purpose of this paper is to prove an analogue of Madsen's conjecture. To motivate our result, we note that Madsen's conjecture can be reduced to the conjecture that the mod 2 unstable Hurewicz map of Coker $J$ detects precisely the Kervaire invariant one elements and nothing else. This reduction follows from the Quillen-Tornehave splitting $Q_{0} S^{0} \simeq$ Coker $J \times \operatorname{Im} J$ (cf. [May]; see [Min5, §5] for a discussion), with $\operatorname{Im} J$ well understood [Qui2], [Sul], [May], [Mah4]. Then our main result, Theorem 4.1, is that the integral Hurewicz image of the spectrum coker $j$, whose associated infinite loop space is Coker $J$, detects precisely the Kervaire invariant one elements and nothing else.
\end{abstract}

1991 Mathematics Subject Classification: Primary 55Q10, 55Q45, 55T15; Secondary 55R12, 55R35, 55N22, 57R77.

Key words and phrases: Adams-Novikov spectral sequence, BP-theory, transfer, stable homotopy groups of the sphere, Kahn-Priddy theorem, the Kervaire invariant one element, image $J$ spectrum. 
Our approach consists of a study of the $j$-Hurewicz image of the $\overline{H \mathbb{Z}}_{(p)}$ (§3) and a "trick" of a commutative diagram (see the proof of Theorem 4.1). The former gives us a very short proof of the Barratt-Jones-Mahowald conjecture [BJM2] concerning the $j$-Hurewicz image of $\mathbb{R} P^{\infty}$, which was also proved by Knapp [Kna]. While Knapp's approach is longer for the Barratt-Jones-Mahowald conjecture itself, we may interpret a pay-off of Knapp's approach, using our "trick" (see Theorem 4.5(ii)).

Our results and approach were announced in [Min1].

The author would like to express his gratitude to Karlheinz Knapp and Erich Ossa for their hospitality during his visits to the University of Wuppertal.

Also, the author would like to express his gratitude to the referee for his careful reading of a preliminary version of this paper.

Notations and conventions. As usual, we set $q=2(p-1)$, and $\mathbb{Z} / p\{g\}$ stands for a cyclic group with $g$ as its generator. For simplicity, we set $P=\Sigma^{\infty} B \Sigma_{p} . H \mathbb{Z} / p_{*}$ and $H \mathbb{Z}_{(p)_{*}}$ stand for the $\bmod p$ and $\bmod \mathbb{Z}_{(p)}$ homology theories, respectively.

$B P$ is the Brown-Peterson spectrum [BP], [Qui1] with

$$
B P_{*}=\mathbb{Z}_{(p)}\left[v_{1}, v_{2}, \ldots\right],
$$

where $v_{n}$ is the Hazewinkel generator [Haz]. For a multi-index $I=\left(i_{1}, \ldots, i_{n}\right)$, we write $|I|=i_{1}+\ldots+i_{n}$ and $v^{I}=v_{1}^{i_{1}} \ldots v_{n}^{i_{n}} . B P\langle 1\rangle$ is the JohnsonWilson spectrum [JW] with $B P\langle 1\rangle_{*}=\mathbb{Z}_{(p)}\left[v_{1}\right]$, which is equipped with the canonical map $\varrho: B P \rightarrow B P\langle 1\rangle$ characterized in homotopy by $v_{1} \mapsto v_{1}$, $v_{i} \mapsto 0$ if $i \geq 2$.

In the following, $k$ is chosen to be 3 (resp. to generate $\left.\left(\mathbb{Z} / p^{2}\right)^{*}\right)$ if $p=2$ (resp. if $p$ is odd), and $\psi^{k}$ is the corresponding Adams operation (cf. [Nov], [Ara]). For $E=B P$ or $B P\langle 1\rangle, J_{E}=$ fiber of $\psi^{k}-1: E \rightarrow E$.

Furthermore, we set

$$
\begin{aligned}
j \mathbb{C} & =\text { fiber of } \psi^{k}-1: B P\langle 1\rangle \rightarrow \Sigma^{q} B P\langle 1\rangle, \\
j & = \begin{cases}\text { fiber of } \psi^{k}-1: b o \rightarrow \Sigma^{4} b s p & \text { if } p=2, \\
j \mathbb{C} & \text { if } p>2 .\end{cases}
\end{aligned}
$$

These various $J$ spectra are related by the following commutative diagrams of cofiber sequences:

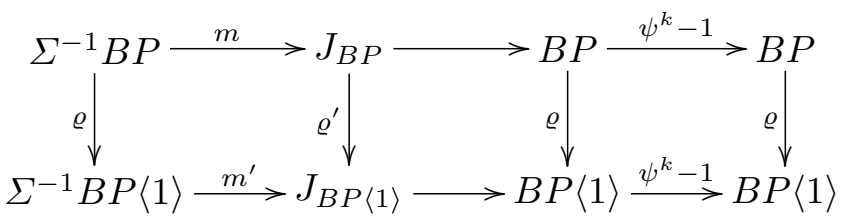




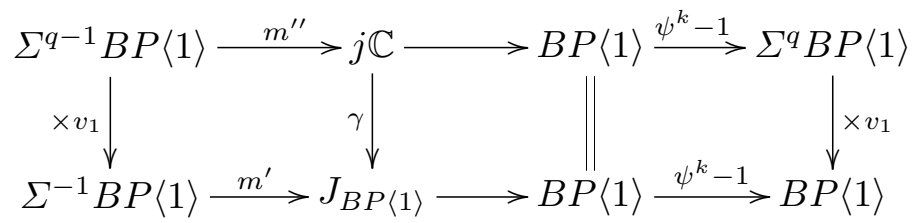

Let $E$ be a spectrum with a specified "unit" map $S^{0} \rightarrow E$ (e.g. a ring spectrum). Then we denote the fiber of $S^{0} \rightarrow E$ by $\bar{E}$. However, for $j=E$, we write

$$
\text { coker } j=\bar{j}
$$

to emphasize that it is the cokernel $J$ spectrum. For an arbitrary spectrum $X$, we write

$$
H_{E}: \pi_{*}(X) \rightarrow E_{*}(X)
$$

for the stable $E$-Hurewicz map induced by $S^{0} \rightarrow E$, and $E_{2}^{s, t}(X, E)=E_{2}$ for the second term of the Adams spectral sequence based on $E$ abutting to $\pi_{t-s}(X)$.

When $E=B P$, we may make the usual homological algebra interpretation [Rav3]:

$$
\begin{gathered}
\operatorname{Ext}_{B P_{*} B P}^{s, t}\left(B P_{*}, B P_{*}(X)\right)=E_{2}^{s, t}(X, B P), \\
P B P_{*}(X)=\operatorname{Ext}_{B P_{*} B P}^{0, *}\left(B P_{*}, B P_{*}(X)\right) .
\end{gathered}
$$

Furthermore, for a $B P_{*} B P$-comodule $M$, we may use either one of

$$
H^{s, t}(M)=\operatorname{Ext}^{s, t}(M)
$$

to stand for $\operatorname{Ext}_{B P_{*} B P}^{s, t}\left(B P_{*}, M\right)$, when there is no danger of confusion.

2. Chromatic spectral sequence. We first recall the fundamental concept of the chromatic spectral sequence due to Miller-Ravenel-Wilson [MRW]. Set $N^{0}:=B P_{*}$; define $B P_{*} B P$-comodules $N^{n}$ and $M^{n}$ inductively by the short exact sequence $0 \rightarrow N^{n} \rightarrow M^{n} \rightarrow N^{n+1} \rightarrow 0$, where $M^{n}=$ $v_{n}^{-1} B P_{*} \otimes_{B P_{*}} N^{n}$. By the standard argument, these short exact sequences give us the chromatic spectral sequence converging to $\operatorname{Ext}\left(B P_{*}\right)$ with $E_{1}^{n, s}=$ $\operatorname{Ext}^{s}\left(M^{n}\right)$.

As noticed by Ravenel [Rav4], [Rav2], the chromatic spectral sequence may be realized geometrically. Set $N_{0}:=S^{0}$; define spectra $N_{n}$ and $M_{n}$ inductively by the cofiber sequence $N_{n} \rightarrow M_{n} \rightarrow N_{n+1}$, where $M_{n}=L_{n} N_{n}$. (Here $L_{n}$ is the Bousfield localization [Bou] with respect to the spectrum $v_{n}^{-1} B P$.) Applying $B P_{*}(-)$ to this cofiber sequence, we recover the short exact sequence above.

We now recall the determination of $\operatorname{Ext}^{0}\left(N^{2}\right)$ by Miller-Ravenel-Wilson [MRW, Th. 6.1, L. 7.2] (for $p$ odd) and Shimomura [Shi, Th. 3.4] (for $p=2$ ). 
TheOREM 2.1 [MRW]. For an odd prime $p, \operatorname{Ext}^{0}\left(N^{2}\right)$ is the direct sum of cyclic p-groups generated by

(1) (a) $x_{2, i} /\left(p v_{1}^{j}\right)$ for $i \geq 0, j \geq 1$ such that $j \leq p^{i}$ and either $p \nmid j$ or $a_{2, i-1}<j$;

(b) $x_{2, i} /\left(p^{k+1} v_{1}^{j}\right)$ for $i \geq 0, j \geq 1, k \geq 1$ such that $p^{k} \mid j \leq a_{2, i-k}$ and either $p^{k+1} \nmid j$ or $a_{2, i-k-1}<j$;

(c) $x_{2, i}^{s} /\left(p^{k+1} v_{1}^{j}\right)$ for $p \nmid s \geq 2, i \geq 0, j \geq 1, k \geq 0$, such that $p^{k} \mid j \leq$ $a_{2, i-k}$ and either $p^{k+1} \nmid j$ or $a_{2, i-k-1}<j$; and

(2) $1 /\left(p^{k+1} v_{1}^{j}\right)$ for $k \geq 0, p^{k} \mid j$, and $j \geq 1$.

Here, $a_{2, i}$ 's are defined by $a_{2,0}=1, a_{2, i}=p^{i}+p^{i-1}-1$ for $i \geq 1$; and $x_{2, i}$ 's are defined inductively by $x_{2,0}=v_{2}, x_{2,1}=v_{2}^{p}-v_{1}^{p} v_{2}^{-1} v_{3}, x_{2,2}=x_{2,1}^{p}-$ $v_{1}^{p^{2}-1} v_{2}^{p^{2}-p+1}-v_{1}^{p^{2}+p-1} v_{2}^{p^{2}-2 p} v_{3}, x_{2, i}=x_{2, i-1}^{p}-2 v_{1}^{(p+1)\left(p^{i-1}-1\right)} v_{2}^{(p-1) p^{i-1}+1}$ for $i \geq 3$.

Theorem 2.2 [Shi]. For $p=2, \operatorname{Ext}^{0}\left(N^{2}\right)$ is the direct sum of cyclic 2-groups generated by

(1) (a) $v_{2}^{s} /\left(2 v_{1}\right)$ for $s$ odd and $\geq 1$;

(b) $x_{2,1}^{s} /\left(2 v_{1}^{j}\right)$ for $s$ odd and $\geq 1$, and $j=1$ or 2 ;

(c) $x_{2,2} /\left(2 v_{1}^{k}\right)$ for $k=1,3,4$;

(d) $x_{2,2}^{s} /\left(2 v_{1}^{k}\right)$ for $s$ odd and $\geq 3$, and $k=1,3,4,5$, or 6 ;

(e) $x_{2,2} /\left(4 x_{1,1}\right)$; and

(f) $x_{2,2}^{s} /\left(8 x_{1,1}\right)$ for $s$ odd and $\geq 3$;

(2) (a) $x_{2, i} /\left(2 v_{1}^{j}\right)$ for $i \geq 3, j \leq 2^{i}$, and either $j$ is odd or $a_{2, i-1}<j$; and

(b) $x_{2, i}^{s} /\left(2 v_{1}^{j}\right)$ for $s$ odd and $\geq 3, i \geq 3, j \leq a_{2, i}$, and either $j$ is odd or $a_{2, i-1}<j$;

(3) $x_{2, i}^{s} /\left(2^{k+1} v_{1}^{j 2^{k}}\right)$ for $s$ odd and $\geq 1, j, k \geq 1, i \geq 3$, and $a_{2, i-k-1}<$ $j 2^{k} \leq a_{2, i-k}$

(4) $x_{2, i}^{s} /\left(2^{k+2} x_{1, k}^{j}\right)$ for $s$ odd and $\geq 1, i \geq 3, k \geq 1, j$ odd and $\geq 1$, and $j 2^{k} \leq a_{2, i-k-1} ;$ and

(5) $1 /\left(2 v_{1}^{j}\right), 1 /\left(2^{k+2} x_{1, k}^{j}\right)$ for $j$ odd and $\geq 1$ and $k \geq 1$.

Here, $a_{2, i}$ 's are defined by $a_{2,0}=1, a_{2,1}=2, a_{2, i}=3 \cdot 2^{i-1}$ for $i \geq 2$; $x_{2, i}$ 's are defined inductively by $x_{2,0}=v_{2}, x_{2,1}=v_{2}^{2}-v_{1}^{2} v_{2}^{-1} v_{3}, x_{2,2}=$ $x_{2,1}^{2}-v_{1}^{3} v_{2}^{3}-v_{1}^{5} v_{3}, x_{2, i}=x_{2, i-1}^{2}$ for $i \geq 3$; and $x_{1, i}$ 's are defined inductively by $x_{1,0}=v_{1}, x_{1,1}=v_{1}^{2}+4 v_{1}^{-1} v_{2}, x_{1, i}=x_{1, i-1}^{2}$ for $i \geq 2$.

For our purpose, we must slightly modify the chromatic object. We first recall (cf. [Rav3, p. 188]) that the $I_{\infty}$-adic filtration for elements in $M^{n}$ or 
$N^{n}$ is defined by

$$
\frac{v^{I}}{v^{J}} \in F^{k} \Leftrightarrow|I|-|J| \geq k
$$

Then, just as before, we have a short exact sequence

$$
0 \rightarrow F^{k} N^{n} \rightarrow F^{k} M^{n} \rightarrow F^{k} N^{n+1} \rightarrow 0
$$

for any $k \in \mathbb{Z}$ and $n \geq 0$. Notice that the case $k=n=0$ is induced by the cofiber sequence $S^{0} \rightarrow H \mathbb{Z}_{(p)} \rightarrow \Sigma \overline{H \mathbb{Z}}_{(p)}$, because $B P_{*} H \mathbb{Z}_{(p)}=$ $\mathbb{Z}_{(p)}\left[v_{1} / p, v_{2} / p, \ldots\right]$. By an analogy with the construction of $N_{2}$, we define $\widetilde{N}_{2}$ to be the cofiber of $\overline{H \mathbb{Z}}_{(p)} \rightarrow L_{1} \overline{H \mathbb{Z}}_{(p)}$, which leads to a commutative diagram of cofiber sequences

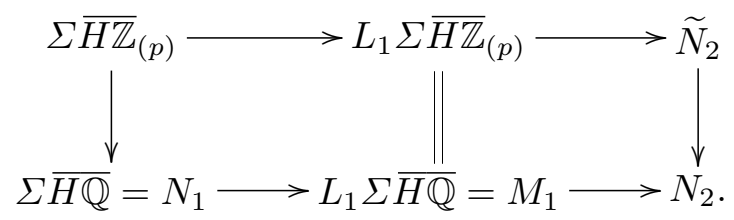

Here the right hand side vertical map induces a surjective $B P_{*} B P$-comodule map $e: \widetilde{N}^{2} \rightarrow N^{2}$, where $\widetilde{N}^{2}=B P_{*}\left(\widetilde{N}_{2}\right)$ and $N^{2}=B P_{*}\left(N_{2}\right)$.

We now analyze the corresponding algebraic situation in detail.

Lemma 2.3. Consider a commutative diagram of exact sequences

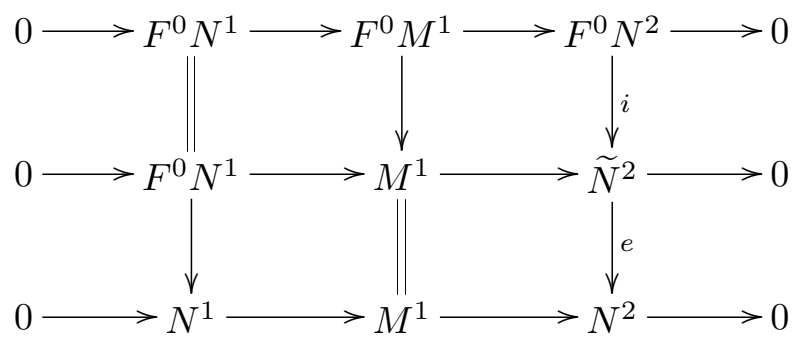

where unnamed maps are the obvious ones, $i$ and $e$ are the induced maps, and the composite $e \circ i$ is the canonical inclusion of the filtration. Then:

(i) $e_{*}: H^{0, t}\left(\widetilde{N}^{2}\right) \rightarrow H^{0, t}\left(N^{2}\right)$ is an isomorphism, except for $t=0$.

(ii) For $t>0$, the inclusion $i_{*}: H^{0, t}\left(F^{0} N^{2}\right) \rightarrow H^{0, t}\left(\widetilde{N}^{2}\right)$ is onto except for the elements which are sent to one of $x_{2, i} /\left(p v_{1}^{p^{i}}\right) \in \operatorname{Ext}\left(N^{2}\right)$ by the isomorphism $e_{*}$.

Proof. (i) It suffices to show that $H^{s, t}(\operatorname{Ker} e)=0$ except for $s=t=0$. For this, note that Ker $e \cong N^{1} / F^{0} N^{1} \cong M^{0} / F^{0} M^{0}$, where the latter isomorphism follows from $F^{0} N^{0}=N^{0}=B P_{*}$. However, $F^{0} M^{0}=B P_{*}\left(H \mathbb{Z}_{(p)}\right)$ and $M^{0}=B P_{*}(H \mathbb{Q})$, where both $H \mathbb{Z}_{(p)}$ and $H \mathbb{Q}$ are $B P$-module spectra. 
Thus, $H^{s, t}\left(F^{0} M^{0}\right) \cong H^{s, t}\left(M^{0}\right) \cong 0$, except for $s=t=0$. Now the claim follows immediately.

(ii) Having established (i), this follows immediately by looking at the $I_{\infty}$-adic filtration of the generators of $\operatorname{Ext}^{0}\left(N^{2}\right)$, which can be read off from Theorems 2.1 and 2.2. Just notice that all the positive dimensional elements listed in Theorem 2.1 and Theorem 2.2 are contained in $F^{0} N^{2}$ except $x_{2, i} /\left(p v_{1}^{p^{i}}\right)$ (which in turn implies $x_{2, i} /\left(p v_{1}^{p^{i}}\right)$ is not hit because of the left exactness of $H^{0}$ ). (We do not have to consider Theorems 2.1(2) and 2.2(5), because they are in the negative dimensions.) Similar analysis showed up during the study of the Thom reduction ([Rav3, 5.4.6]).

REMARK 2.4. The previous lemma distinguishes $x_{2, j} /\left(p v_{1}^{p^{j}}\right)$, which essentially corresponds to the Kervaire invariant one elements for $j \geq 1$ (resp. $j \geq 0$ ) when $p=2$ (resp. $p$ odd) [Rav1], [Rav3]. For this, we first note that, from Theorems 2.1 and 2.2 , we can easily see (cf. [Rav3, 5.4.6])

$$
H^{0, t}\left(N^{2}\right)=(e \circ i)_{*} H^{0, t}\left(F^{0} N^{2}\right) \oplus \mathbb{Z} / p\left\{\frac{x_{2, j}}{p v_{1}^{p^{j}}}\right\},
$$

where the second factor shows up only if $t=q p^{j+1}$. Then, as discussed in [Rav3, 5.4.6], the following composite of the boundary homomorphisms and the Thom reduction

$$
\begin{aligned}
H^{0, q p^{j+1}}\left(N^{2}\right) \rightarrow H^{1, q p^{j+1}}\left(N^{1}\right) \rightarrow H^{2, q p^{j+1}}\left(N^{0}\right) & \\
& =\operatorname{Ext}_{B P_{*} B P}^{2, q p^{j+1}}\left(B P_{*}, B P_{*}\right) \rightarrow \operatorname{Ext}_{\mathcal{A}_{*}^{2, q p^{j+1}}}(\mathbb{Z} / p, \mathbb{Z} / p)
\end{aligned}
$$

is characterized by the following two properties:

(1) $(e \circ i)_{*} H^{0, q p^{j+1}}\left(F^{0} N^{2}\right)$ goes to 0 ;

(2) $x_{2, j} /\left(p v_{1}^{p^{j}}\right)$ goes to $h_{j+1}^{2}$ (resp. $\left.-b_{j}\right)$ if $p=2$ (resp. if $p$ is odd).

Furthermore, if there is a Kervaire invariant one element in $\pi_{q p^{j+1}-2}^{s}\left(S^{0}\right)$ for $j \geq 1$ (resp. $j \geq 0$ ) when $p=2$ (resp. $p$ odd), then it is detected in $H^{0, q p^{j+1}}\left(N^{2}\right)$ because such an element should come from $\pi_{*}^{s}(\operatorname{coker} j)$. From this discussion, any element in $H^{0, q p^{j+1}}\left(N^{2}\right) \backslash(e \circ i)_{*} H^{0, q p^{j+1}}\left(F^{0} N^{2}\right) \cong$ $H^{0, q p^{j+1}}\left(\widetilde{N}^{2}\right) \backslash i_{*} H^{0, q p^{j+1}}\left(F^{0} N^{2}\right)$ may be called a Kervaire invariant one element.

For our purpose, $x_{2, j} /\left(p v_{1}^{p^{j}}\right)$ is rather difficult to deal with because of the complicated definition of $x_{2, j}$. Now the following lemma provides us with a simpler substitute.

Lemma 2.5. (i) (cf. [Rav1]) $v_{2}^{p^{j}} /\left(p v_{1}^{p^{j}}\right) \in F^{-1} \backslash F^{0}$ is primitive in $N^{2}$, but not so in $\widetilde{N}^{2}$. 
(ii) $v_{2}^{p^{j}} /\left(p v_{1}^{p^{j}}\right)-v_{1}^{p^{j+1}} /\left(p^{p^{j+1}+1}\right) \in F^{-1} \backslash F^{0}$ is primitive in $\tilde{N}^{2}$ and sent to $v_{2}^{p^{j}} /\left(p v_{1}^{p^{j}}\right)$ by the isomorphism $e_{*}$.

Proof. (i) Since $\eta_{R}\left(v_{2}\right) \equiv v_{2}+v_{1} t_{1}^{p}-v_{1}^{p} t_{1} \bmod p$, we have

$$
\eta_{R}\left(v_{2}^{p^{j}}\right) \equiv v_{2}^{p^{j}}+v_{1}^{p^{j}} t_{1}^{p^{j+1}}\left(\bmod \left(p, v_{1}^{p^{j+1}}\right)\right) .
$$

Also, since $\eta_{R}\left(v_{1}\right)=v_{1}+p t_{1}, \eta_{R}\left(v_{1}^{-1}\right) \equiv v_{1}^{-1}(\bmod p)$. Thus,

$$
\eta_{R}\left(\frac{v_{2}^{p^{j}}}{p v_{1}^{p^{j}}}\right) \equiv \frac{v_{2}^{p^{j}}}{p v_{1}^{p^{j}}}+\frac{t_{1}^{p^{j+1}}}{p},
$$

where $t_{1}^{p^{j+1}} / p=0$ in $N^{2}$, but not so in $\widetilde{N}^{2}$.

(ii) Since $\eta_{R}\left(v_{1}\right)=v_{1}+p t_{1}$, we have

$$
\eta_{R}\left(v_{1}^{p^{j+1}}\right) \equiv v_{1}^{p^{j+1}}+p^{p^{j+1}} t_{1}^{p^{j+1}},
$$

modulo elements in filtration $\geq p^{j+1}+1$. Thus,

$$
\eta_{R}\left(\frac{v_{1}^{p^{j+1}}}{p^{p^{j+1}+1}}\right) \equiv \frac{v_{1}^{p^{j+1}}}{p^{p^{j+1}+1}}+\frac{t_{1}^{p^{j+1}}}{p},
$$

from which the claim follows.

Finally, we have arrived at the main result of this section:

Corollary 2.6. For $t>0$, we have

$$
H^{0, t}\left(\widetilde{N}^{2}\right)=i_{*} H^{0, t}\left(F^{0} N^{2}\right) \oplus \mathbb{Z} / p\left\{\frac{v_{2}^{p^{j}}}{p v_{1}^{p^{j}}}-\frac{v_{1}^{p^{j+1}}}{p^{p^{j+1}+1}}\right\},
$$

where the second factor shows up only if $t=q p^{j+1}$.

3. $H_{j}: \pi_{*}\left(\overline{H \mathbb{Z}}_{(p)}\right) \rightarrow j_{*}\left(\overline{H \mathbb{Z}}_{(p)}\right)$. Consider the following commutative diagram of cofiber sequences:

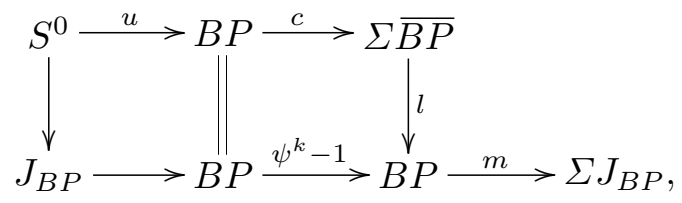

where $u$ is the unit map, $c$ is the induced cofiber map, $m$ is the cofiber map induced by $\psi^{k}-1$, and the induced map $l$ factorizes as $\Sigma \overline{B P} \stackrel{u \wedge 1}{\longrightarrow}$ $B P \wedge \Sigma \overline{B P} \stackrel{l^{\prime}}{\rightarrow} B P$. From this, we see $\psi^{k}-1$ factorizes as

$$
B P \stackrel{d_{1}}{\rightarrow} B P \wedge \Sigma \overline{B P} \stackrel{l^{\prime}}{\rightarrow} B P,
$$


where $d_{1}:=(u \wedge 1) \circ c$ induces the first differential in the canonical $B P$ based Adams-Novikov spectral sequence. This fact clearly indicates that $m \circ l^{\prime}$ induces a natural transformation

$$
L: \operatorname{Ext}^{1}\left(B P_{*}(X)\right) \rightarrow J_{B P}(X)
$$

and we have a commutative diagram

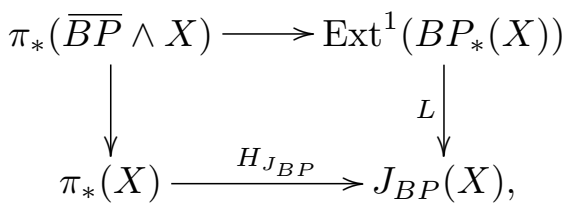

where the unnamed maps are the obvious ones.

LEMMA 3.1. Let $W \stackrel{f}{\rightarrow} X \stackrel{g}{\rightarrow} Y \stackrel{h}{\rightarrow} \Sigma W$ be a cofiber sequence with $B P_{*}(h)=0$. Then we have a commutative diagram

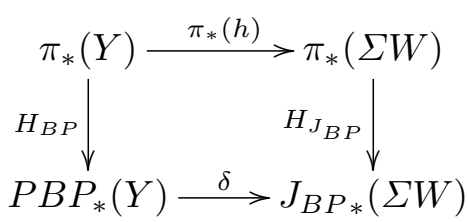

where $\delta$ is the composition of the connecting homomorphism associated with

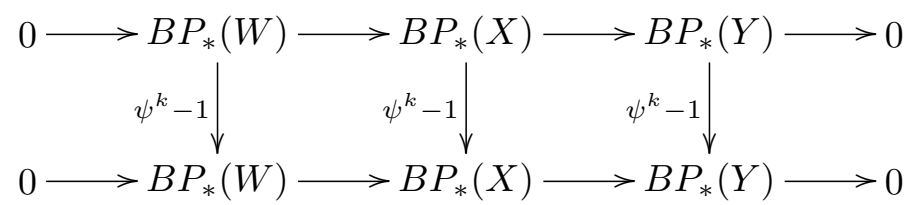

and the canonical map $B P_{*}(W) \rightarrow J_{B P_{*}}(\Sigma W)$ induced by $m: \Sigma^{-1} B P$ $\rightarrow J_{B P}$.

Proof. Let $\alpha \in \pi_{*}(Y)$. Set $a:=H_{B P}(\alpha) \in P B P_{*}(Y) \subseteq \operatorname{Ker}\left(\psi^{k}-1\right)$. Then, since $B P_{*}(h)=0$, the geometric connecting homomorphism theorem ([JMWZ], [Rav, 2.3.4]) implies that $\pi_{*}(h)(\alpha)$ is detected in $B P_{*}(\Sigma W)$ by $\delta^{\prime}(a)$, where $\delta^{\prime}$ is the connecting homomorphism associated with

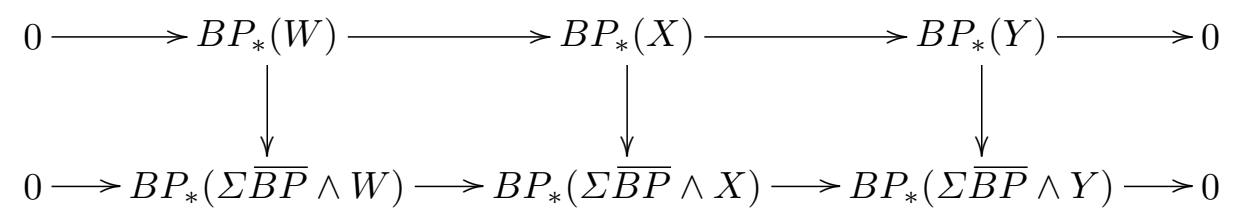


where vertical maps are induced by $d_{1}$. Since $\psi^{k}-1=l^{\prime} \circ d_{1}$, the claim follows.

Proposition 3.2. Suppose $t>0$. Then the composite

$$
H^{0, t}\left(\widetilde{N}^{2}\right)=P B P_{t}\left(\widetilde{N}_{2}\right) \stackrel{\delta}{\rightarrow} J_{B P t-1}\left(\Sigma \overline{H \mathbb{Z}}_{(p)}\right) \stackrel{\varrho^{\prime}}{\rightarrow} J_{B P\langle 1\rangle_{t-1}}\left(\Sigma \overline{H \mathbb{Z}}_{(p)}\right)
$$

is characterized by:

(i) The precomposition with $i_{*}: H^{0, t}\left(F^{0} N^{2}\right) \rightarrow H^{0, t}\left(\widetilde{N}^{2}\right)$ is trivial.

(ii) $v_{2}^{p^{j}} /\left(p v_{1}^{p^{j}}\right)-v_{1}^{p^{j+1}} /\left(p^{p^{j+1}+1}\right)$ goes non-trivially to an order $p$ element in $J_{B P\langle 1\rangle_{q p^{j+1}-2}}\left(\overline{H \mathbb{Z}}_{(p)}\right)$.

P r o o f. We first examine this composite $\varrho^{\prime} \circ \delta$ in detail. Let $x \in H^{0, t}\left(\widetilde{N}^{2}\right)$. We can define a canonical lift $\widetilde{x} \in M^{1}$ with respect to the short exact sequence $0 \rightarrow F^{0} N^{1} \rightarrow M^{1} \rightarrow \widetilde{N}^{2} \rightarrow 0$. In fact, we can do so by regarding our representatives of $H^{0, t}\left(\widetilde{N}^{2}\right)(t \neq 0)$, given by Theorems $2.1,2.2$, and Corollary 2.6, as elements of $M^{1}$. Then calculate $\left(\psi^{k}-1\right) \widetilde{x}$, which is $\left(k^{n}-1\right) \widetilde{x}$ if $x \in$ $H^{0,2 n}\left(\widetilde{N}^{2}\right)$ [Ara]. This element turns out to be in $F^{0} N^{1}=B P_{*}\left(\Sigma \overline{H Z}_{(p)}\right)$. Now the desired value $\varrho^{\prime} \circ \delta(x)$ is calculated as the image of $\left(\psi^{k}-1\right) \widetilde{x}$ under the composite $B P_{t}\left(\Sigma \overline{H Z}_{(p)}\right) \stackrel{\varrho}{\rightarrow} B P\langle 1\rangle_{t}\left(\Sigma \overline{H Z}_{(p)}\right) \stackrel{m^{\prime}}{\rightarrow} J_{B P\langle 1\rangle_{t-1}}\left(\Sigma \overline{H \mathbb{Z}}_{(p)}\right)$, where we have used the notations and the commutativity of the diagram at the end of Section 1. We also notice that

$$
\begin{aligned}
B P_{*}\left(\Sigma \overline{H Z}_{(p)}\right) & \cong\left(\mathbb{Z}_{(p)}\left[v_{1} / p, v_{2} / p, \ldots\right]\right) /\left(\mathbb{Z}_{(p)}\left[v_{1}, v_{2}, \ldots\right]\right), \\
B P\langle 1\rangle_{*}\left(\Sigma \overline{H Z}_{(p)}\right) & \cong\left(\mathbb{Z}_{(p)}\left[v_{1} / p\right]\right) /\left(\mathbb{Z}_{(p)}\left[v_{1}\right]\right) \oplus(\text { sum of } \mathbb{Z} / p \text { 's })
\end{aligned}
$$

(cf. $[\mathrm{MM}])$, and $\varrho: B P_{*}\left(\Sigma \overline{H \mathbb{Z}}_{(p)}\right) \rightarrow B P\langle 1\rangle_{*}\left(\Sigma \overline{H \mathbb{Z}}_{(p)}\right)$ is characterized by $v_{1} / p \mapsto v_{1} / p, v_{l} / p \mapsto 0(l \geq 2)$.

For (i), the claim follows from the fact that every positive-dimensional element in $H^{0, t}\left(\widetilde{N}^{2}\right)$ that is in the image of $i_{*}$ is $i_{*}$ of a fraction that become 0 when $v_{l}$ is set to 0 for $l \geq 2$ (cf. Theorems 2.1 and 2.2.). In fact, if $x$ has such a property, then $\left(\psi^{k}-1\right) \widetilde{x}$ has the same property. Thus, $\varrho\left(\left(\psi^{k}-1\right) \widetilde{x}\right)=0$, which verifies the claim.

For (ii), we notice

$$
B P\langle 1\rangle_{q p^{j+1}-1}\left(\overline{H Z}_{(p)}\right)=\mathbb{Z} / p^{p^{j+1}}\left\{\left(\frac{v_{1}}{p}\right)^{p^{j+1}}\right\} \oplus(\text { sum of } \mathbb{Z} / p \text { 's }),
$$

and choose $e$ so that $k^{(p-1) p^{j+1}}-1=u \cdot p^{e}$ with $p \nmid u$. Then $p^{j+1}-e \geq 0$, because $e=j+3$ (resp. $e=j+2$ ) if $p=2$ (resp. if $p$ is odd) [Ada2, (2.12)]. Now let

$$
x=\frac{v_{2}^{p^{j}}}{p v_{1}^{p^{j}}}-\frac{v_{1}^{p^{j+1}}}{p^{p^{j+1}+1}} .
$$


Then

$$
\varrho\left(\psi^{k}-1\right) \widetilde{x}=-u \cdot \frac{v_{1}^{p^{j+1}}}{p^{p^{j+1}-e+1}}
$$

becomes a nonzero element of order $p^{p^{j+1}-e+1}$ in $B P\langle 1\rangle_{q p^{j+1}-1}\left(\overline{H \mathbb{Z}}_{(p)}\right)$. Thus, to show that $\varrho^{\prime} \circ \delta(x)$ is a nontrivial element of order $p$, it suffices to show that $-u \cdot v_{1}^{p^{j+1}} / p^{p^{j+1}-e+1}$ is not in the image of $\psi^{k}-1$, in view of the exact sequence

$$
B P\langle 1\rangle_{q p^{j+1}-1}\left(\overline{H \mathbb{Z}}_{(p)}\right) \stackrel{\psi^{k}-1}{\longrightarrow} B P\langle 1\rangle_{q p^{j+1}-1}\left(\overline{H \mathbb{Z}}_{(p)}\right) \stackrel{m^{\prime}}{\rightarrow} J_{B P\langle 1\rangle_{q p^{j+1}-2}}\left(\overline{H \mathbb{Z}}_{(p)}\right) .
$$

For this purpose, since $\psi^{k}-1$ acts on $\mathbb{Z} / p^{p^{j+1}} \subset B P\langle 1\rangle_{q p^{j+1}-1}\left(\overline{H \mathbb{Z}}_{(p)}\right)$ as multiplication by $u \cdot p^{e}$ (recall that this cyclic group is the image of $\varrho$ ), it suffices to show $\psi^{k}$ respects the direct sum decomposition $B P\langle 1\rangle_{*}\left(\Sigma \overline{H \mathbb{Z}}_{(p)}\right)$ $\cong \mathbb{Z}_{(p)}\left[v_{1} / p\right] / \mathbb{Z}_{(p)}\left[v_{1}\right] \oplus$ (sum of $\mathbb{Z} / p$ 's $)$. However, this immediately follows by noticing that the canonical map

$$
B P\langle 1\rangle_{*}\left(\Sigma \overline{H Z}_{(p)}\right) \rightarrow E(1)_{*}\left(\Sigma \overline{H Z}_{(p)}\right)
$$

commutes with $\psi^{k}$, maps the factor $\mathbb{Z}_{(p)}\left[v_{1} / p\right] / \mathbb{Z}_{(p)}\left[v_{1}\right]$ injectively, and kills the other factor consisting of $\mathbb{Z} / p$ 's.

Remark 3.3. (i) Proposition 3.2 immediately solves the Barratt-JonesMahowald Conjecture [BJM2], which claims any lift of a Kervaire invariant one element is detected by the $j$-Hurewicz map $H_{j}: \pi_{*}(P) \rightarrow j_{*}(P)$.

(ii) We have another similar decomposition of $B P\langle 1\rangle_{*}\left(\Sigma \overline{H \mathbb{Z}}_{(p)}\right)$. In fact, let $\lambda: P \rightarrow \overline{H \mathbb{Z}}_{(p)}$ be the lift of a Kahn-Priddy map, then $B P\langle 1\rangle_{*}(\lambda)$ becomes injective and induces a direct sum decomposition

$$
B P\langle 1\rangle_{*}\left(\overline{H \mathbb{Z}}_{(p)}\right) \cong B P\langle 1\rangle_{*}(P) \oplus(\text { sum of } \mathbb{Z} / p \text { 's }) .
$$

By exactly the same argument as the one given at the end of the proof of Proposition 3.2(ii), we find $\psi^{k}$ respects this decomposition. In particular, this implies $J_{B P\langle 1\rangle_{*}}(\lambda): J_{B P\langle 1\rangle_{*}}(P) \rightarrow J_{B P\langle 1\rangle_{*}}\left(\overline{H \mathbb{Z}}_{(p)}\right)$ is an embedding.

Proposition 3.4. For $*>0$, the composite

$$
\pi_{*}\left(S^{0}\right) \cong \pi_{*}\left(\overline{H \mathbb{Z}}_{(p)}\right) \stackrel{H_{j \mathbb{C}}}{\longrightarrow} j \mathbb{C}\left(\overline{H \mathbb{Z}}_{(p)}\right)
$$

detects precisely the $B P_{*}$-Adams-Novikov filtration one elements and the Kervaire invariant one elements, and nothing else.

Proof. We first prove the same detection property for the composite

$$
H_{J_{B P\langle 1\rangle}}: \pi_{*}\left(\overline{H \mathbb{Z}}_{(p)}\right) \stackrel{H_{j \mathbb{C}}}{\longrightarrow} j \mathbb{C}_{*}\left(\overline{H \mathbb{Z}}_{(p)}\right) \stackrel{\gamma}{\rightarrow} J_{B P\langle 1\rangle_{*}}\left(\overline{H Z}_{(p)}\right) .
$$

For this, applying Lemma 3.1 to the cofiber sequence $\Sigma \overline{H \mathbb{Z}}_{(p)} \rightarrow L_{1} \Sigma \overline{H \mathbb{Z}}_{(p)}$ $\left(=M_{1}\right) \rightarrow \widetilde{N}_{2} \rightarrow \Sigma\left(\Sigma \overline{H Z}_{(p)}\right)$, we get the following commutative diagram: 


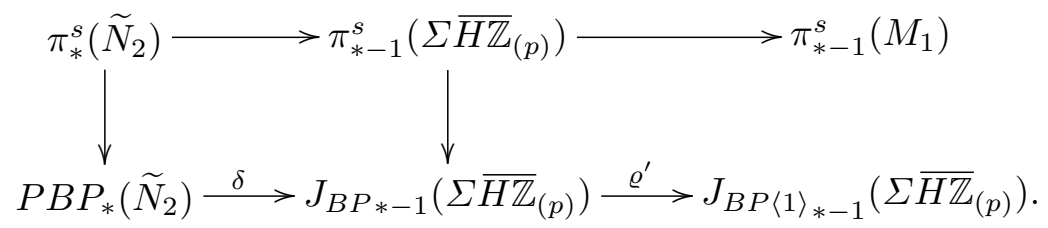

From this, Remark 2.4, and Proposition 3.2, all the $v_{1}$-torsion elements, i.e. those annihilated in $\pi_{*-1}^{s}\left(M_{1}\right)$, which are detected in $J_{B P\langle 1\rangle_{*-1}}\left(\Sigma \overline{H \mathbb{Z}}_{(p)}\right)$ are precisely the Kervaire invariant one elements in $\pi_{q p^{j+1}-2}^{s}\left(S^{0}\right)$ for $j \geq 1$ (resp. $j \geq 0$ ) when $p=2$ (resp. $p$ odd), and nothing else. Thus, it suffices to show that the set of the $B P$-Adams filtration 1 permanent cycle elements is the same as, possibly modulo Kervaire invariant one elements, the set of $v_{1}$-local elements, i.e. those detected in $\pi_{*}\left(M_{1}\right)$, which are detected in $J_{B P\langle 1\rangle_{*-1}}\left(\Sigma \overline{H Z}_{(p)}\right)$. (When $p=2$, it is easy to check the Kervaire invariant one element $\eta^{2} \in \pi_{2}^{s}\left(S^{0}\right)$ is detected in $j \mathbb{C}\left(\overline{H \mathbb{Z}}_{(p)}\right)$.)

Notice that this is trivial when $p$ is odd, since all the $v_{1}$-local elements are of $B P$-Adams filtration 1 and they are all detected in $B P\langle 1\rangle_{*-1}\left(\Sigma \overline{H \mathbb{Z}}_{(p)}\right)$.

However, we have to work extra for $p=2$, because there are $v_{1}$-periodic elements of $B P$-Adams filtration $\geq 2$. Since no element of $B P$-Adams filtration $\geq 3$ is detected in $J_{B P *-1}\left(\Sigma \bar{H}_{\mathbb{Z}}(p)\right.$ ) (cf. the diagram just before Lemma 3.1 ), it suffices to show no $v_{1}$-periodic element of $B P$-Adams filtration 2 is detected in $J_{B P\langle 1\rangle_{*-1}}\left(\Sigma \overline{H \mathbb{Z}}_{(p)}\right)$. Then, as is well known ([Rav3, 5.1]), any $v_{1}$-periodic $B P$-Adams filtration 2 (permanent cycle) element is of the form $\eta p_{2 n-1}$, where $\eta \in \pi_{1}^{S}\left(S^{0}\right)$ is the usual generator and $p_{2 n-1} \in \operatorname{Ext}^{1,2 n}\left(B P_{*}\right)$ is some $B P$-Adams permanent cycle. Therefore, together with the KahnPriddy theorem, it suffices to show that the $\eta$ action on $J_{B P\langle 1\rangle_{2 n-1}}(P)$ is trivial for relevant $n$.

For this purpose, we remark that, given $p \in J_{B P\langle 1\rangle_{2 n-1}}(P), \eta p \in$ $J_{B P\langle 1\rangle_{2 n}}(P)$ is calculated via the geometric boundary theorem associated with $S^{1} \stackrel{\eta}{\rightarrow} S^{0} \rightarrow S^{0} \cup_{\eta} e^{2} \rightarrow S^{2}$, smashed with $P$. In fact, let $B P\langle 1\rangle_{*}\left(S^{0} \cup_{\eta}\right.$ $\left.e^{2}\right)=B P\langle 1\rangle_{*}\left\{x_{0}, x_{2}\right\}$, where the generators $x_{0} \in B P\langle 1\rangle_{0}, x_{2} \in B P\langle 1\rangle_{2}$ are chosen so that $\psi^{3} x_{2}=x_{2}+v_{1} x_{0}$. Now recall $\left.\psi^{3}\right|_{B P\langle 1\rangle_{2 n-1}(P)}=$ multiplication by $3^{n}$ (cf. the proof of Proposition 3.2). Then, regarding $x_{2} \otimes p \in$ $B P\langle 1\rangle_{*}\left(\left(S^{0} \cup_{\eta} e^{2}\right) \wedge P\right)$ and using $\left(\psi^{3}-1\right)(p)=0$, we calculate

$$
\begin{aligned}
\left(\psi^{3}-1\right)\left(x_{2} \otimes p\right) & =\left(\psi^{3} x_{2}\right) \otimes\left(\psi^{3} p\right)-x_{2} \otimes p=\left(x_{2}+v_{1} x_{0}\right) \otimes\left(\psi^{3} p\right)-x_{2} \otimes p \\
& =x_{2} \otimes\left(\psi^{3}-1\right) p+x_{0} \otimes\left(3^{n} v_{1} p\right)=x_{0} \otimes\left(3^{n} v_{1} p\right),
\end{aligned}
$$

from which we see

$$
\eta p=m^{\prime}\left(3^{n} v_{1} p\right),
$$

where $m^{\prime}: B P\langle 1\rangle_{2 n+1}(P) \rightarrow J_{B P\langle 1\rangle_{2 n}}(P)$. Thus, to show $\eta p=0$, it suffices to show $v_{1} p \in\left(\psi^{3}-1\right)\left(B P\langle 1\rangle_{2 n+1}(P)\right)$. 
This is certainly the case for $n$ even, since $v_{1} p \in v_{1} B P\langle 1\rangle_{2 n-1}(P)=$ $2 B P\langle 1\rangle_{2 n+1}(P)$ and $\left.\left(\psi^{3}-1\right)\right|_{B P\langle 1\rangle_{2 . \text { odd }-1}(P)}=2 \cdot($ odd $)$, where we have used

$$
\nu_{2}\left(3^{n}-1\right)= \begin{cases}\nu_{2}(n)+2 & \text { if } n \text { is even, } \\ 1 & \text { if } n \text { is odd. }\end{cases}
$$

For $n$ odd, $\left.\left(\psi^{3}-1\right)\right|_{B P\langle 1\rangle_{2 \cdot \text { odd }-1}}=2 \cdot($ odd) implies $2 p=0$, and so $2 v_{1} p=0$ in $B P\langle 1\rangle_{2 n+1}(P)$. Since $B P\langle 1\rangle_{2 n+1}(P) \cong \mathbb{Z} / 2^{n+1}$, we see $v_{1} p \in$ $2^{n} B P\langle 1\rangle_{2 n+1}(P) \subset B P\langle 1\rangle_{2(n+1)-1}(P)$. So this element is in the $\left(\psi^{3}-1\right)$ image as far as

$$
n \geq \nu_{2}(n+1)+2 .
$$

This is certainly the case for any odd $n \geq 5$. Furthermore, the cases $n=3$ and $n=1$ are irrelevant, for $\pi_{5}^{s}\left(S^{0}\right) \cong 0$ and $\pi_{2}^{s}\left(S^{0}\right) \cong \mathbb{Z} / 2$ is generated by the Kervaire invariant one element $\theta_{1}$.

Thus, the above discussion completes the proof of the detection property of the composite

$$
\pi_{*}\left(S^{0}\right) \cong \pi_{*}\left(\overline{H \mathbb{Z}}_{(p)}\right) \stackrel{H_{j \mathbb{C}}}{\longrightarrow} j \mathbb{C}_{*}\left(\overline{H \mathbb{Z}}_{(p)}\right) \stackrel{\gamma}{\rightarrow} J_{B P\langle 1\rangle_{*}}\left(\overline{H \mathbb{Z}}_{(p)}\right) .
$$

Finally, to complete the proof of Proposition 3.4, we must show $\gamma$ is injective on the image of $H_{j \mathbb{C}}$. For this, consider the following commutative diagram:

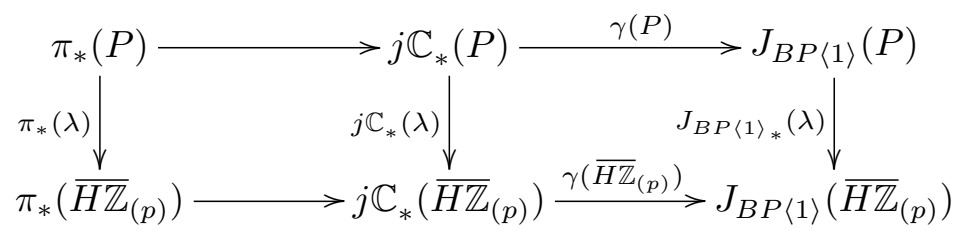

Since $\pi_{*}(\lambda)$ is surjective by the Kahn-Priddy theorem $[\mathrm{KP}]$ and $J_{B P\langle 1\rangle_{*}}(\lambda)$ is injective by Remark 3.3(ii), it suffices to show $\gamma(P)$ is injective. But, this immediately follows from the following commutative diagram of exact sequences:

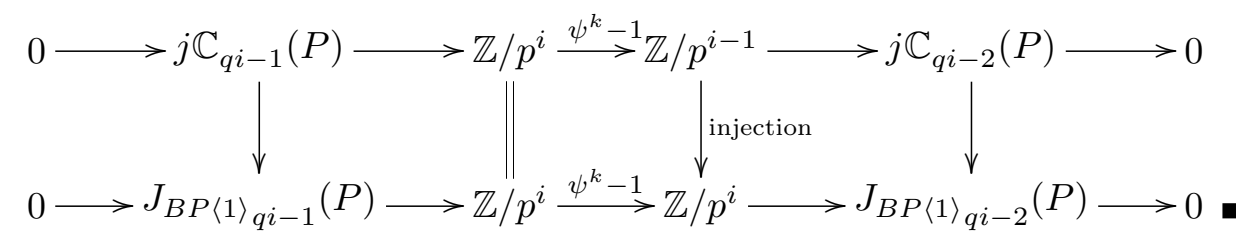

For our purpose, we need to prove a statement for the $j$-Hurewicz map, rather than the $j \mathbb{C}$-Hurewicz map. With Proposition 3.4 at hand, we may restrict ourselves to the case $p=2$. Now, up to the Barratt-Jones-Mahowald conjecture, the fundamental work of Mahowald [Mah4, Th. 7.10] implies, when $p=2, H_{j}: \pi_{*}(P) \rightarrow j_{*}(P)$ detects precisely the $v_{1}$-local elements, i.e. the image $J$ related elements, the Kervaire invariant one elements, and 
some Kahn-Priddy lifts of the Mahowald $\eta_{j}$-elements (we call any image $J$ unrelated element in $\pi_{2^{j}}^{s}\left(S^{0}\right)$ detected by $h_{1} h_{j}$ a Mahowald $\eta_{j}$-element, which exists by [Mah3]; we also assume $j \geq 4$, for otherwise it would become an image $J$ related element), and nothing else.

Thus, we must determine the fate of the image of the Mahowald $\eta_{j^{-}}$ elements under the map $j_{*}(\lambda): j_{*}(P) \rightarrow j_{*}\left(\overline{H Z}_{(2)}\right)$. For this, recall from [Mah4, pp. 97-98] that, for $j \geq 3, j_{2^{j}}(P) \cong \mathbb{Z} / 2 \oplus \mathbb{Z} / 2$, where the first factor gives the stable Hopf invariant $\nu$ and the second factor corresponds to the Adams $\mu_{2^{j}}$-element. When we consider the composite $j_{2^{j}}(P) \rightarrow$ $j_{2^{j}}\left(\overline{H \mathbb{Z}}_{(2)}\right) \rightarrow j_{2^{j}}\left(S^{0}\right) \cong \mathbb{Z} / 2$, the second factor goes isomorphically to the target, while the first factor is annihilated. Note that from our assumption that $\eta_{j}$ is not detected in $\pi_{*}^{s}\left(S^{0}\right)$ by the image of $J$, if any of its Kahn-Priddy lifts is detected in $j_{2^{j}}(P)$, then the image should be the generator of the first factor. Let us denote this generator of the first factor by $g_{j} \in j_{2^{j}}(P)$. We now prove the following:

Lemma 3.5. (i) For any $j$, there is a choice of $\eta_{j}$ which has two distinct Kahn-Priddy lifts $k_{j}$ and $l_{j}$ such that $H_{j}\left(k_{j}\right)=g_{j}$ and $H_{j}\left(l_{j}\right)=0$, where $H_{j}: \pi_{*}(P) \rightarrow j_{*}(P)$.

(ii) No $\eta_{j}$ is detected by $\pi_{*}\left(S^{0}\right) \cong \pi_{*}\left(\overline{H \mathbb{Z}}_{(2)}\right) \stackrel{H_{j}}{\rightarrow} j_{*}\left(\overline{H \mathbb{Z}}_{(2)}\right)$.

Proof. (i) (cf. [CLM]) As was discussed in [CJM, (4.10)], there exists a Kahn-Priddy lift $l_{j}$ of some $\eta_{j}$ such that $l_{j}: S^{2^{j}} \rightarrow P^{2^{j-1}} \subset P$ is detected by the functional $\mathrm{Sq}^{2^{j}}$-operation. So, this element is detected by $h_{1} h_{j}$ in the Adams spectral sequence of the sphere and, for dimensional reasons, this element does not have $\nu$ as its stable Hopf invariant. Thus, by adding $\mu_{2^{j}}$ if necessary to make it image $J$ unrelated, we may assume $l_{j}$ is a Kahn-Priddy lift of some $\eta_{j}$ such that $H_{j}\left(l_{j}\right)=0$.

To construct $k_{j}$, we recall [Seg], [Kuh] that an inverse of the Kahn-Priddy map may be provided by the composite $Q_{0} S^{0} \stackrel{s}{\simeq} Q_{0} S^{0} \stackrel{J H}{\rightarrow} Q B \mathbb{Z} / 2$, where $J H$ is the James-Hopf map associated with the Kahn-Snaith splitting [Kah], [Sna] and $s$ is a self homotopy equivalence used to make $\lambda \circ J H \circ s$ homotopic to the identity. Now, we set $k_{j}: S^{2^{j}} \rightarrow P$ to be the lift of $\eta_{j}$ (which is the Kahn-Priddy image of $l_{j}$ as above), obtained by applying $J H \circ s$ to (the unstable adjoint of) $\eta_{j}$. Since the James-Hopf invariant converts the EHPsequence to the stable $E H P$-sequence [CMT], [Kuh], the claim follows from [Mah1] (see [Min3] for a simpler treatment of this fact), which claims that the Hopf invariant of any $\eta_{j}$ is $\nu$. We note that from the image $J$ unrelated assumption on $\eta_{j}, k_{j}$ is detected by $g_{j}$, the generator of the first factor of $j_{2^{j}}(P) \cong \mathbb{Z} / 2 \oplus \mathbb{Z} / 2$, as was discussed just prior to Lemma 3.5.

(ii) Let $\widetilde{\eta}_{j}$ be any $\eta_{j}$, i.e. an image $J$ unrelated element detected by $h_{1} h_{j}$, and let $p_{j}$ be its Kahn-Priddy lift. If $H_{j}\left(p_{j}\right)=0$ then the claim is 
trivial; we may assume $H_{j}\left(p_{j}\right) \neq 0$. Then, from part (i) and the explanation just before Lemma $3.5, H_{j}\left(p_{j}\right)=g_{j}=H_{j}\left(k_{j}\right)$. This implies the composite $\pi_{*}\left(S^{0}\right) \cong \pi_{*}\left(\overline{H \mathbb{Z}}_{(p)}\right) \stackrel{H_{j}}{\rightarrow} j_{*}\left(\overline{H \mathbb{Z}}_{(p)}\right)$ sends $\lambda\left(p_{j}\right)=\widetilde{\eta}_{j}$ and $\lambda\left(k_{j}\right)=\eta_{j}$ to the same element. However, $\eta_{j}$ is annihilated by this composite for $\eta_{j}=\lambda\left(l_{j}\right)$ and $H_{j}\left(l_{j}\right)=0$. Thus, $\widetilde{\eta}_{j}$ is also annihilated as desired.

Now, we have the main result of this section.

TheOREM 3.6. For $*>0$, the composite

$$
\pi_{*}\left(S^{0}\right) \stackrel{\cong}{\rightarrow} \pi_{*}\left(\overline{H Z}_{(p)}\right) \stackrel{H_{j}}{\rightarrow} j_{*}\left(\overline{H \mathbb{Z}}_{(p)}\right)
$$

detects precisely the image $J$ related elements and the Kervaire invariant one elements, and nothing else.

Proof. The claim for the case $p>2$ follows from Proposition 3.4. For the case $p=2$, by the above result of Mahowald [Mah4] and Lemma 3.5, this composite detects at most the image $J$ related elements and the Kervaire invariant one elements. Of course, the image $J$ related elements are detected because they are the elements detected in $j_{*}\left(S^{0}\right)$ (via the map $j_{*}\left(\overline{H Z}_{(p)}\right) \rightarrow$ $\left.j_{*}\left(S^{0}\right)\right)$. On the other hand, the Kervaire invariant one elements are detected by Proposition 3.2 (see Remark 3.3(i)). Thus the claim follows.

\section{Stable Hurewicz image of the cokernel $J$ spectrum}

THEOREM 4.1. $H_{\mathbb{Z}_{(p)}}: \pi_{*}($ coker $j) \rightarrow\left(H \mathbb{Z}_{(p)}\right)_{*}($ coker $j)$ detects precisely the Kervaire invariant one elements in $\pi_{q p^{j+1}-2}^{s}\left(S^{0}\right)$ for $j \geq 1$ (resp. $j \geq 0$ ) when $p=2$ (resp. $p$ odd $)$, and nothing else.

Proof. Let $i$ : coker $j \rightarrow S^{0}$ and $i^{\prime}: \overline{H \mathbb{Z}}_{(p)} \rightarrow S^{0}$ be the fiber of the unit maps of $j$ and $H \mathbb{Z}_{(p)}$, respectively. Then, in view of Theorem 3.6, it suffices to show, for $x \in \pi_{*}(\operatorname{coker} j)(*>0)$,

$$
H_{\mathbb{Z}_{(p)}}(x) \neq 0 \Leftrightarrow H_{j}\left(i_{*}^{\prime-1}\left(i_{*}(x)\right)\right) \neq 0 .
$$

However, since $i_{*}$ is injective, this easily follows from the following commutative diagram of exact sequences:

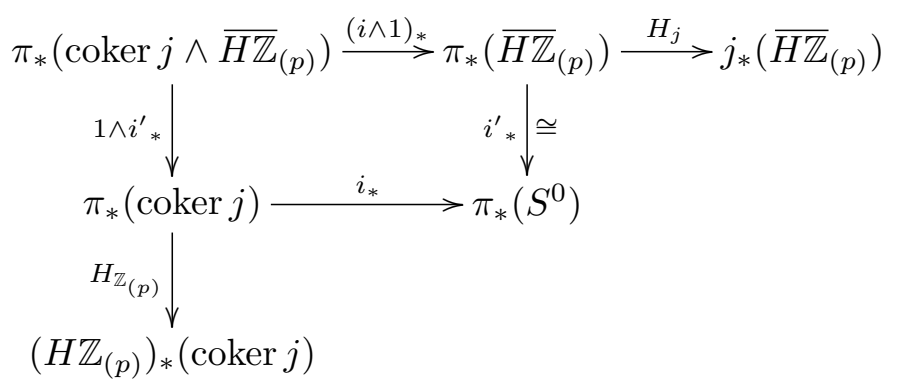


As was mentioned in Section 1, this gives an analogue of Madsen's conjecture about the unstable Hurewicz image of $Q_{0} S^{0}$, when $p=2$. For $p \geq 5$, Theorem 4.1 and the result of Ravenel [Rav1] implies the stable Hurewicz image is nontrivial only for the bottom class element which corresponds to $\beta_{1}\left(=\theta_{0}\right.$ for $p$ odd $)$.

Corollary 4.2. For $p \geq 5, H_{\mathbb{Z}_{(p)}}: \pi_{*}(\operatorname{coker} j) \rightarrow\left(H \mathbb{Z}_{(p)}\right)_{*}(\operatorname{coker} j)$ detects only the bottom class element, i.e. a nonzero multiple of $\beta_{1}$.

Actually, such a property is available for any prime $p$, if we consider the $\bmod p$ Hurewicz map instead.

Theorem 4.3. $H_{\mathbb{Z} / p}: \pi_{*}(\operatorname{coker} j) \rightarrow(H \mathbb{Z} / p)_{*}($ coker $j)$ detects only the bottom class element, i.e. $\nu^{2}$ (resp. a nonzero multiple of $\left.\beta_{1}\right)$ if $p=2$ (resp. if $p$ is odd).

Proof. Since we may apply the same argument as the one given in the proof of Theorem 4.1, it suffices to show that the obvious map $j_{q p^{j+1}-2}\left(\overline{H \mathbb{Z}}_{(p)}\right) \rightarrow j_{q p^{j+1}-2}\left(\overline{H \mathbb{Z}}_{(p)} p\right)$ kills higher Kervaire invariant one elements. Here, by higher Kervaire invariant one element, we mean $\theta_{j}$ for $j \geq 4$ (resp. $j \geq 1$ ) when $p=2$ (resp. when $p$ is odd). For this, consider the following commutative diagram of cofiber sequences:

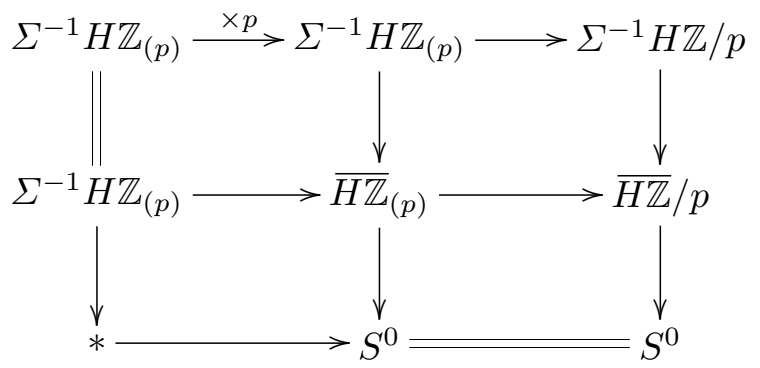

First, we claim that the middle upper vertical map induces an isomorphism

$$
j_{q p^{j+1}-2}\left(\Sigma^{-1} H \mathbb{Z}_{(p)}\right) \cong j_{q p^{j+1}-2}\left(\overline{H Z}_{(p)}\right) .
$$

In fact, this follows from $j_{q p^{j+1}-2}\left(S^{0}\right)=0$ and the surjectivity of $j_{*}\left(\overline{H \mathbb{Z}}_{(p)}\right)$ $\rightarrow j_{*}\left(S^{0}\right)(*>0)$, which is induced by the middle lower vertical map.

Then, from an easy diagram chase, it suffices to show that the higher Kervaire invariant one elements are detected in $j_{q p^{j+1}-2}\left(\overline{H Z}_{(p)}\right)$ by a $p$ divisible element. But this is actually the case for $p=2$ by [Mah4, p. 98], which claims that the image of $H_{j}: \pi_{2^{j+1}-2}^{s}(P) \rightarrow j_{2^{j+1}-2}(P) \cong \mathbb{Z} / 2^{j}$ is contained in $\mathbb{Z} / 2\left(\subseteq \mathbb{Z} / 2^{j}\right)$ for $j \geq 1$. For $p$ odd, we can easily check this by slightly modifying the calculation in the proof of Proposition 3.2(ii), using the last commutative diagram in the proof of Proposition 3.4. 
From the proof of Theorem 4.3, we see that for any $n$ the $\bmod p^{n}$ Hurewicz image of cokernel $J$ spectrum detects just finitely many Kervaire invariant one elements and nothing else.

In [Kna], Knapp has also proved the Barratt-Jones-Mahowald conjecture by a different method. To motivate his approach, we notice that, for $* \neq 0, \pi_{*}(\overline{B P\langle 1\rangle}) \cong \pi_{*}\left(S^{0}\right) \oplus \pi_{*+1}(B P\langle 1\rangle)$, where $\pi_{*}\left(S^{0}\right)$ is embedded as the torsion part of $\pi_{*}(\overline{B P\langle 1\rangle})$; we let $t: \pi_{*}\left(S^{0}\right) \rightarrow \pi_{*}(\overline{B P\langle 1\rangle})$ be the corresponding embedding. Now the main result of Knapp is the following.

Theorem 4.4 [Kna]. For $*>1$, the composite

$$
\pi_{2 *-2}\left(S^{0}\right) \stackrel{t}{\rightarrow} \pi_{2 *-2}(\overline{B P\langle 1\rangle}) \stackrel{H_{j \mathbb{C}}}{\longrightarrow} j \mathbb{C}_{2 *-2}(\overline{B P\langle 1\rangle})
$$

detects precisely the Kervaire invariant one elements and nothing else.

Warning: In [Kna], $\bar{E}$ stands for the cofiber of the unit map, not the fiber of the unit map. Of course, our convention, including Theorem 4.4, stands for the latter.

Knapp's approach is a more complicated way of proving the BarrattJones-Mahowald conjecture, but his result (quoted as Theorem 4.4) gives an interesting corollary using a diagram like the one in the proof of Theorem 4.1 .

TheOrem 4.5. Suppose $p$ is odd. Then we have

(i) The BP-Hurewicz map of the cokernel $J$ spectrum detects precisely the Adams-Novikov 2-line elements and nothing else.

(ii) The $B P\langle 1\rangle$ Hurewicz image of the cokernel $J$ spectrum detects precisely the Kervaire invariant one elements and nothing else.

Proof. (i) First, we claim that, for any $x \in \pi_{n}(\operatorname{coker} j)$,

$$
H_{B P}(x) \neq 0 \Leftrightarrow H_{j}\left(t\left(i_{*}(x)\right)\right) \neq 0 .
$$

In fact, this immediately follows from the analogous commutative diagram of exact sequences as the one in the proof of Theorem 4.1, because $\pi_{n+1}$ (coker $j$ $\wedge \overline{B P}$ ) is torsion. Next, since $p$ is odd, any $x \in \pi_{n}$ (coker $j$ ) is detected in the $B P$-Adams-Novikov (for $\pi_{*}\left(S^{0}\right)$ ) 2-line or higher. Now the claim follows immediately, as the Hattori-Stong theorem [Hat], [Sto] implies

$$
\operatorname{Ext}_{B P_{*} B P}^{2, *}\left(B P_{*}, B P_{*}\right)=\operatorname{Ext}_{B P_{*} B P}^{1, *}\left(B P_{*}, B P_{*}(\overline{B P})\right) \rightarrow j \mathbb{C}_{*}(\overline{B P})
$$

is injective, for $\overline{B P}$ is torsion free (cf. [Kna]).

(ii) This is proved exactly as (i), using Knapp's result (Theorem 4.4). 


\section{References}

[Ada1] J. F. Adams, On the non-existence of elements of Hopf invariant one, Ann. of Math. 72 (1960), 20-104.

[Ada2] -, On the groups $J(X)$ - II, Topology 3 (1965), 137-171.

[Ara] S. Araki, Multiplicative operations in BP cohomology, Osaka J. Math. 12 (1975), 343-356.

[BJM1] M. G. Barratt, J. D. S. Jones and M. E. Mahowald, The Kervaire invariant problem, in: Contemp. Math. 19, Amer. Math. Soc., 1983, 9-23.

[BJM2] -, - - - The Kervaire invariant and the Hopf invariant, Lecture Notes in Math. 1286, Springer, 1987, 135-173.

[Bou] A. K. Bousfield, The localization of spectra with respect to homology, Topology 18, (1979), 257-281.

[Bro] W. Browder, The Kervaire invariant of a framed manifold and its generalization, Ann. of Math. 90 (1969), 157-186.

[BP] E. H. Brown and F. P. Peterson, A spectrum whose $\mathbb{Z} / p$ cohomology is the algebra of reduced $p$-th powers, Topology 5 (1966), 149-154.

[CJM] R. L. Cohen, J. D. S. Jones and M. E. Mahowald, The Kervaire invariant of immersions, Invent. Math. 79 (1985), 95-123.

[CMT] F. R. Cohen, J. P. May and L. R. Taylor, Splitting certain spaces CX, Math. Proc. Cambridge Philos. Soc. 84 (1978), 465-496.

[CLM] R. L. Cohen, W.-H. Lin and M. E. Mahowald, The Adams spectral sequence of the real projective spaces, Pacific J. Math. 134 (1988), 27-55.

[CJK] M. C. Crabb, S. Jäschke and K. Knapp, The $\operatorname{Im}(J)$-theory Chern character, preprint.

[Cur] E. B. Curtis, The Dyer-Lashof algebra and the $\Lambda$-algebra, Illinois J. Math. 19 (1975), 231-246.

[Hat] A. Hattori, Integral characteristic numbers for weakly almost complex manifolds, Topology 5 (1966), 259-280.

[Haz] M. Hazewinkel, Formal Groups and Applications, Academic Press, New York, 1978.

[JMWZ] D. C. Johnson, H. R. Miller, W. S. Wilson and R. S. Zahler, Boundary homomorphisms in the generalized Adams spectral sequence and the non-triviality of infinitely many $\gamma_{t}$ in stable homotopy, in: Reunion sobre teorie de homotopia, Soc. Mat. Mexicana, 1975, 47-59.

[JW] D. C. Johnson and W. S. Wilson, Projective dimension and Brown-Peterson homology, Topology 12 (1973), 327-353.

[Kah] D. S. Kahn, On the stable decomposition of $\Omega^{\infty} S^{\infty}$ A, Lecture Notes in Math. 658, Springer, 1978, 206-214.

[KP] D. S. Kahn and S. B. Priddy, Applications of the transfer to stable homotopy theory, Bull. Amer. Math. Soc. 78 (1972), 981-987.

[Ker] M. A. Kervaire, A manifold which does not admit any differentiable structure, Comment. Math. Helv. 34 (1960), 256-270.

[KM] M. A. Kervaire and J. W. Milnor, Groups of homotopy spheres: I, Ann. of Math. 77 (1963), 504-537.

[Kna] K. Knapp, $\operatorname{Im}(J)$-theory and the Kervaire invariant, preprint.

[Kuh] N. J. Kuhn, The geometry of James-Hopf maps, Pacific J. Math. 102 (1982), 397-412.

[Liu] A. L. Liulevicius, The factorization of cyclic reduced power by secondary cohomology operations, Mem. Amer. Math. Soc. 42 (1962). 
[Mad] I. Madsen, On the action of Dyer Lashof algebra in $H_{*}(G)$ and $H_{*}(G / T O P)$, Dissertation, University of Chicago, 1970.

[Mah1] M. E. Mahowald, Some Whitehead products in $S^{n}$, Topology 4 (1965), 17-26.

[Mah2] - Some remarks on the Kervaire invariant problem from the homotopy point of view, in: Proc. Sympos. Pure Math. 22, Amer. Math. Soc., 1971, 165-169.

[Mah3] -, A new infinite family in ${ }_{2} \pi_{*}^{s}$, Topology 16 (1977), 249-256.

[Mah4] - , The image of $J$ in the EHP sequence, Ann. of Math. 116 (1982), 65-112.

[MM] M. E. Mahowald and R. J. Milgram, Operations that detect $\mathrm{Sq}^{4}$ in connective K-theory and their applications, Quart. J. Math. Oxford 27 (1976), 415-432.

[May] J. P. May (with contributions by F. Quinn, N. Ray, and J. Tornehave), $E_{\infty}$ Ring Spaces and $E_{\infty}$ Ring Spectra, Lecture Notes in Math. 577, Springer, 1977.

[MRW] H. R. Miller, D. C. Ravenel and W. S. Wilson, Periodic phenomena in the Adams-Novikov spectral sequence, Ann. of Math. 106 (1977), 469-516.

[Min1] N. Minami, On the Hurewicz image of the cokernel $J$ spectrum, Abstracts Amer. Math. Soc. 16 (1995), 897-55-783.

[Min2] —, The Kervaire invariant one element and the double transfer, Topology 34 (1995), 481-488.

[Min3] -, On the odd primary Adams 2-line elements, preprint.

[Min4] - , The iterated transfer analogue of the new doomsday conjecture, Trans. Amer. Math. Soc., to appear.

[Min5] -, An introduction to the Kervaire invariant one problem, in: Proc. Sympos. Pure Math., to appear.

[Nov] S. P. Novikov, The methods of algebraic topology from the viewpoint of cobordism theories, Izv. Akad. Nauk SSSR Ser. Mat. 31 (1967), 855-951 (in Russian); English transl.: Math. USSR-Izv. (1967), 827-913.

[Qui1] D. G. Quillen, On the formal group laws of unoriented and complex cobordism theory, Bull. Amer. Math. Soc. 75 (1969), 1293-1298.

[Qui2] -, The Adams conjecture, Topology 10 (1971), 1-10.

[Rav1] D. C. Ravenel, The nonexistence of odd primary Arf invariant elements in stable homotopy, Math. Proc. Cambridge Philos. Soc. 83 (1978), 429-443.

[Rav2] -, Localization with respect to certain periodic homology theories, Amer. J. Math. 106 (1984), 351-414.

[Rav3] - Complex Cobordism and Stable Homotopy Groups of Spheres, Academic Press, Orlando, Fla., 1986.

[Rav4] - The geometric realization of the chromatic resolution, in: Algebraic Topology and Algebraic K-Theory, Ann. of Math. Stud. 113, Princeton Univ. Press, Princeton, N.J., 1987, 168-179.

[Seg] G. B. Segal, Operations in stable homotopy theory, in: London Math. Soc. Lecture Note Ser. 11, Cambridge Univ. Press, 1974, 105-110.

[Shi] K. Shimomura, Novikov's Ext $^{2}$ at the prime 2, Hiroshima Math. J. 11 (1981), 499-513.

[Sna] V. P. Snaith, Stable decomposition of $\Omega^{n} \Sigma^{n} X$, J. London Math. Soc. 7 (1974), $577-583$

[Sto] R. E. Stong, Relations among characteristic numbers - I, Topology 4 (1965), $267-281$.

[Sul] D. P. Sullivan, Genetics of homotopy theory and the Adams conjecture, Ann. of Math. 100 (1974), 1-79 
[Wel] R. J. Wellington, The unstable Adams spectral sequence for iterated loop spaces, Mem. Amer. Math. Soc. 258 (1982).

The University of Alabama

Mathematics Department

Box 870350

Tuscaloosa, Alabama 35487-0350

U.S.A.

E-mail: norihiko@gp.as.ua.edu, norihiko@euler.math.ua.edu

Received 7 November 1996;

in revised form 2 July 1997 